Documenta Haematologica, volume XXXIV, nr. 1-2, May 2015

\author{
DE DE GRUYTER \\ OPEN \\ G \\ DOI: 10.1515/dch-2015-0005
}

\title{
Quercetin, Menadione, Doxorubicin combination as a potential alternative to Doxorubicin monotherapy of acute lymphoblastic leukemia
}

Ruxandra Irimia ${ }^{1}$, , Ioana Teodora Tofolean ${ }^{1}$, Roxana Gabriela Sandu ${ }^{1}$, Oana Elena Băran ${ }^{1}$, Maria Cătălina Ceaușescu ${ }^{1}$, Vlad Coşoreanu ${ }^{1}$, Maria Teodora Ilie ${ }^{1}$, Ramona Babeş $^{1}$, Constanţa Ganea ${ }^{1}$, Irina Băran ${ }^{1}$

1. University of Medicine and Pharmacy "Carol Davila”, Bucharest, Romania

These authors contributed equally to the work

\begin{abstract}
:
Doxorubicin is a widely used chemotherapeutic drug, effective on patients with acute lymphoblastic leukemia but associated with significant long term cardio-toxicity. Menadione (vitamine K3) and the flavonoid quercetin are known as strong apoptogens in human leukemia Jurkat T cells.

We explored the potential synergic cytotoxic effects of doxorubicin in association with quercetin and Menadione in this cellular model for acute lymphoblastic leukemia.

Cellular viability, apoptosis, necrosis oxidative stress and cellular cycle were determined by flow cytometry utilizing Jurkat lymphoblasts labeled with Annexin V-FITC/7-AAD, $\mathrm{CM}-\mathrm{H}_{2}$ DCFDA/7-AAD and propidium iodide respectively.

Results indicate a dose-dependent oxidative-stress generation, cell cycle arrest and apoptosis induction by doxorubicin alone, correlated with a decrease of the required doses when the anticancer drug was associated with quercetin and menadione, hence supporting the theory of an additive cytotoxic effect on leukemia cells.

Introducing QC-MD combinations in leukemia doxorubicin-based treatment could significantly increase the treatment's efficacy. The main mechanism responsible for this effect appears to be the increase in DOX affinity for DNA, which enables lowering of the therapeutic dose.
\end{abstract}

\section{Key words:}

doxorubicine, quercetine, menadione, acute lymphoblastic leukemia, oxidative stress

*Corresponding author:

Ruxandra Irimia, Carol Davila University of Medicine and Pharmacy, blvd. Eroii Sanitari nr.8, Bucharest, Romania, phone +40748152880 , e-mail: ruxandra_rm @yahoo.com 


\section{Introduction:}

In the past years, a great interest had been focused on the health benefits of natural flavonoids. They are chemical compounds widely found in fruits, leaves and vegetables, responsible for their bright colours, and also well known potent antioxidants. The possible antioxidant effects of flavonoids were suspected after it was shown that a red wine rich Mediterranean diet was associated with a lower cardio-vascular risk. Numerous studies have shown that they possess antiaterogenic-cardioprotective effects and exert anti-tumoral, anti-mutagenic and anti-inflammatory activities.

Quercetin is known to have also pro-oxidative properties under various conditions, and a number of both in vitro and in vivo studies indicated a greater susceptibility of malignant cells to the cytotoxic effect of the flavonoids with respect to their normal counterparts.

Quercetin is one of the best characterized flavonoids, and it is found prevalently in fresh parsley, onions, olives and green salad.

Flavonoids appear to bind to a sugar molecule, and originally it was considered that only free flavonoids could be absorbed in the human intestine.

Quercetin in its native state is usually bound to a glucose or rutinose molecule.

Studies have indicated a superior absorption of the quercetin-glucose complex, compared to quercetin-rutinose, with a 20 times higher peak plasma level reached in 10 times less time from the ingestion, suggesting a small intestine absorption for the first compound and a large intestine post-deglicosilation for the latter.

The main limitation to the clinical use of quercetin is linked to its poor bioavailability and its rapid metabolic conversion in the liver.

Pharmacokinetic studies had shown that cancer-efficient $(>10 \mu \mathrm{M})$ non-toxic plasma levels of quercetin are maintained for about 30 minutes, the average half life being little above 40 minutes.

Liposomal carriers used to deliver quercetin can increase the half-life and the bioavailability of the flavonoid, allowing achieving clinically effective plasma levels in cancer treatment protocols.

Flavonoids have at least 3 anti-oxidant mechanisms as well as the capacity of stimulating the natural protective capacity.

These mechanisms include:

-direct oxygen-reactive species scavenging, through the high hydroxyl-group-reactivity, resulting in the inactivation of radical species according to the formula:

Flavonoid $(\mathrm{OH})+\mathrm{R}->$ Flavonoid $(\mathrm{O})+\mathrm{RH}$.

This mechanism is probably responsible for the anti-anteroclerotic effect -stimulation of the nitric-oxide (NO) production; studies conducted on acute lymphoblastic leukemia cells after quercetin exposure show an increase of the nitricoxide levels, and NO-syntethise inhibition by marcaptoetilguanidine increases the apoptotic leukemic post-antioxidant treatment cells.

The increase in NO production appears to be a protective mechanism at physiological low concentrations, but in high levels it reacts with oxygen-reactive species, forming peroxynitrate, with devastating effects on cell membranes. 
-Fe chelating properties, demonstrated in some in vitro studies on mice erytrocytes after glutathione depletion.

In the absence of flavonoids, significant membrane lipid peroxidation and hemolysis may occur due to ferum release, while association with quercetin can exert protective effects via ferum chelation.

In the acute lymphoblastic leukemia Jurkat $\mathrm{T}$ cell line, quercetin activates the ryanodine receptor/calcium release channel, hence inducing calcium release from the endoplasmic reticulum, acting as a potent apoptogen and also enhancing apoptosis induced by menadione.

Menadione, also known as vitamin K3, is a well-known pro-oxidant agent, with anti-tumoral activity in various cellular lines, including Jurkat T cells.

It is a lipo-soluble synthetic form of vitamin $\mathrm{K}$, which is metabolized in the human body to vitamin $\mathrm{K} 2$.

Recent studies have shown a chemosensitising effect of menadione in different cancer models, as well as a powerful corrector of doxorubicin resistance in leukemia cells. More than half of the intracellular metabolism of menadione is developed through redox cycling, which can generate high quantities of free radicals.

Both menadione and quercetin are known as proapoptotic agents acting via the calciumdependent mitochondrial pathway, promoting $\mathrm{Ca}^{2+}$ release from the endoplasmic reticulum and opening the mitochondrial permeability transition pore, which in turn induce the collapse of the mitochondrial transmembrane potential and the release of the cytocrome $c$ from mitocondria. Cytocrome $c$ is associated with the internal mitochondrial membrane and is an essential component of the electron transport chain. When released into the cytoplasm, it activates the apoptotic protease activation factor-1, thus triggering apoptosis.

Doxorubicin, also known as Adriamycin, is a very broad spectrum chemotherapeutic drug compared to other antineoplastic drugs, first extracted from Streptomyces peucetius in the 1970'.

There are two main mechanisms by which doxorubicin acts as an anticancer drug:

1) intercalation into DNA and disruption of topoisomerase II-mediated DNA repair, and

2) generation of free oxygen radicals, that generate lipid peroxidation, DNA and protein injury, thus triggering the apoptotic pathways.

Doxorubicin enters the cells through passive diffusion, and once it has entered the cell it can bind to the proteasome.

The doxorubicin-proteasome complex is translocated into the nucleus, where it dissociates and intercalates in the DNA, thus inhibiting the cellular metabolism. Doxorubicin molecules that did not intercalate into the DNA stabilize the complex between topoisomerase II and DNA strings, leading to an increase in the damaged genomic material, associated with G2/M arrest and necrosis/apoptosis.

During the intracellular metabolic conversion of doxorubicin, one electron is added to the quinine, leading to the formation of a semiquinone, that converts back to quinine by reducing oxygen to reactive oxygen species leading to cellular oxidative stress. A major limitation in the clinical use of doxorubicin is its cardiotoxicity. 
The cardiotoxic effect is dependent to the cumulative dose and may occur decades after finishing the treatment. There is evidence supporting the existence of two mechanisms of anticancer activity and cardiotoxicity.

It appears that the cardiotoxic effect is determined by iron-release and free radicals

generation. Doxorubicin is reduced to doxorubicinol, an active metabolite which interferes with intracellular iron deposits.

This theory is supported by the fact that iron chelator dexrazoxane is protective against doxorubicin induced toxicity.

In this case, the association of quercetin and its Fe chelating properties could not only improve the therapeutic index of doxorubicin, but also decreased the cardiotoxic effect.

\section{Materials and methods:}

Jurkat cells (clone E6-1, ATCC) were cultured in GLUTAMAX-I and HEPEScontaining RPMI 1640 medium (Invitrogen) supplemented with 10\% FBS, 100 units/ml penicillin and $100 \mathrm{mg} / \mathrm{ml}$ streptomycin, at $37^{\circ} \mathrm{C}$ in a humidified incubator with a $5 \% \mathrm{CO}_{2}$ atmosphere. Menadione sodium bisulphite (Sigma-Aldrich) was dissolved in PBS, whereas doxorubicin (Tocris), dihydrated quercetin (Sigma-Aldrich) and CM-H2DCFDA (Invitrogen) were dissolved in DMSO.

For evaluation of the cell cycle distribution, 106 cells were washed twice in PBS, incubated for $15 \mathrm{~min}$. in propidium iodide PI/RNAse staining buffer (Pharmingen) containing $0.1 \%$ Triton $\mathrm{X}-100$ and $25 \mu \mathrm{M}$ digitonin in the dark at room temperature, and then analyzed on a Beckman Coulter Gallios flow-cytometer. To measure the DNA content, the cells were stained with PI, which has a red fluorescence. For data acquisition and analysis we used the software CellQuest and WinMDI 2.9, together with a Gaussian deconvolution algorithm. Apoptosis was evaluated as the fraction of hypodiploid cells (the sub-G0/G1 cell fraction). The G0/G1, S, and G2/M cell fractions were calculated for the nonapoptotic cell population, by excluding the hypodiploid events from the cell cycle analysis.

For apoptosis/necrosis assessment, 106 cells were washed twice with PBS and double stained with FITC (fluorescein isothiocyanate) conjugated Annexin V (Pharmingen) and PI (Pharmingen), according to the manufacturer's instructions. The samples were analyzed immediately on a Beckman Coulter Gallios flow-cytometer. The excitation wavelength was $488 \mathrm{~nm}$. Emission was recorded in FL1 $(525 \mathrm{~nm}$, bandpass 40 $\mathrm{nm}$ ) for Annexin V-FITC, and FL3 (620 nm, bandpass $30 \mathrm{~nm}$ ) for PI. Data analysis was performed using the software WinMDI 2.9.

It was considered that:

- cells negative for both Annexin V-FITC and PI were considered as living cells - early apoptotic cells were positive for Anexine V-FITC and negative for PI;

- late apoptotic/necrotic cells due to their permeable plasmatic membrane are positive for both dyes.

For oxidative stress evaluation, cells were washed in a standard saline (SS, containing $140 \mathrm{mM} \mathrm{NaCl}, 5 \mathrm{mM} \mathrm{KCl}, 1 \mathrm{mM} \mathrm{CaCl} 2,1 \mathrm{mM} \mathrm{MgCl}_{2}, 20 \mathrm{mM}$ HEPES, 10 $\mathrm{mM}$ glucose, $\mathrm{pH} 7.4 / \mathrm{NaOH}$ ), resuspended in SS containing $0.5 \mu \mathrm{M}$ CM-H2DCFDA (a general oxidative stress indicator that is largely sensitive to $\mathrm{H}_{2} \mathrm{O}_{2}$ and $\mathrm{OH}$, but can also sense peroxynitrite) and incubated for $10 \mathrm{~min}$. at $37^{\circ} \mathrm{C}$ in the dark. Cells were then 
centrifuged and resuspended in $0.1 \mathrm{ml}$ SS containing 7-AAD (7-Aminoactinomycin D; Pharmingen), according to the manufacturer's instructions. After staining for $15 \mathrm{~min}$. at room temperature in the dark, samples were diluted with $0.4 \mathrm{ml} \mathrm{SS}$ and measured immediately on a Beckman Coulter Gallios flow-cytometer. CM-H2DCFDA and 7-AAD emission was recorded on FL1 and FL4 (675 nm, bandpass $20 \mathrm{~nm})$, respectively.

\section{Results:}

Doxorubicin is known to undergo several oxido-reduction processes to form a doxorubicin semiquinone radical, an unstable metabolite which is converted back to doxorubicin in a process that is generally accompanied by a dose-dependent production of free radicals. In agreement with this characteristic, we observed that after exposure for $18 \mathrm{~h}$, DOX induced the production of reactive oxygen species and decreased the viable cell fraction in a dose dependent manner, with a half-maximal inhibitory concentration IC50 $=0.57 \mu \mathrm{M}$ (Fig. $1 \mathrm{~A}$ ). The derived Hill coefficient, $\mathrm{H}=1.44$, indicated that cooperativity of at least two DOX molecules was required to induce the observed cytotoxic effect.

$15 \mu \mathrm{M}$ QC combined with $7.5 \mu \mathrm{M} \mathrm{MD}$ produced $31 \%$ dead cells, with associated oxidative stress and combination with DOX exhibited additive cytotoxicity (IC50 $=2.26$ $\mu \mathrm{M} ; \mathrm{H}=1.73$ ).

The $15 \mu \mathrm{M}$ equimolar QC/MD combination produced by itself a cell death rate of $52 \%$, associated with oxidative stress generation and apoptosis induction, and enhanced DOX cytotoxicity, producing a dramatic decrease in the viable cell fraction (IC50 $=1.25$ $\mu \mathrm{M} ; \mathrm{H}=1.70$ ).

Cell death was assessed by flow cytometry on CM-H2DCFDA/7-AAD double stained cells. CM-H2DCFDA is an indicator of the intracellular level of oxygen reactive species. In Fig. 1B we can see the increase of oxidative stress dose dependently for doxorubicin and also for menadione.

The $15 \mu \mathrm{M}$ MD dose increased the oxidative stress levels by $167 \%$ compared to the 7.5 $\mu \mathrm{M}$ MD dose, showing an additive cytotoxicity in combination cu doxorubicin (Fig. 1B). 

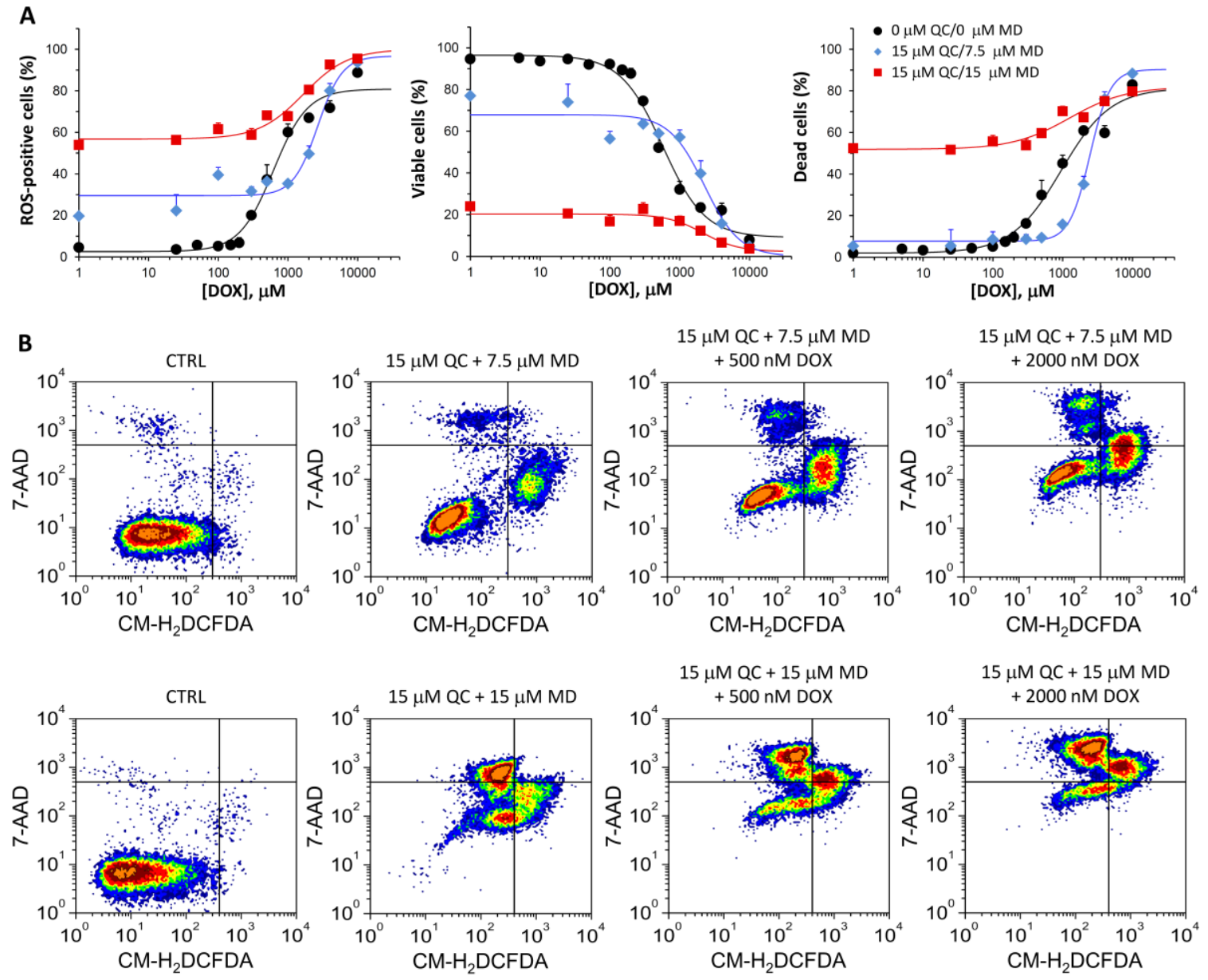

Fig. 1. Oxidative stress generation and cytotoxicity induced by doxorubicin alone or in association with QC and MD at indicated doses for $18 \mathrm{~h}$. (A) Dose-dependent effects of DOX in the absence or presence of QC/MD. (B) Typical bivariate plots obtained by flow cytometry on $\mathrm{CM}-\mathrm{H}_{2} \mathrm{DCFDA} / 7-\mathrm{AAD}$ double stained cells.

On cell cycle, $0.1 \mu \mathrm{M}$ and $1 \mu \mathrm{M}$ DOX selectively arrested the cell cycle in G2/M (63\% cell fraction) and $\mathrm{S}$ phase (70\%), respectively (Figs. 2, 3).

$100 \mathrm{nM}$ DOX combined with $0.1 \mu \mathrm{M}$ QC and $0.1 \mu \mathrm{M}$ MD produced $61 \%$ arrest in $\mathrm{G} 2 / \mathrm{M}$, $29 \%$ in $\mathrm{S}$ phase.

An increase in QC and MD concentration to $1 \mu \mathrm{M}$ determines a decrease of G0/G1 phase arrest, with maintaining of the $\mathrm{S}$ phase percentage and an increase in the pre-replicative percentage of cells.

Addition of QC/MD up to $7.5 \mu \mathrm{M}$ further increased the S-cell fraction as well as the G2/M cell-fraction. Higher levels progressively increased the G0/G1 cell fraction (Figs. 2, 3).

The $1000 \mathrm{nM}$ DOX concentration induced a $73 \%$ S-phase arrest with a dramatic increase in the cellular DNA S-phase peak, and a sub-G0/G1 peak suggesting an increase in the number of apoptotic cells.

Addition of $5 \mu \mathrm{M}$ QC/MD decreased the pre-replicative percentage. 
An increase in the QC and MD concentration led to a peak of S-phase cell percentage at a concentration of $2.5 \mu \mathrm{M}$, associated with a minimal G0/G1 arrest, followed by a decrease in the S-phase cell fraction at higher concentrations with an increase in the G0/G1 phase.
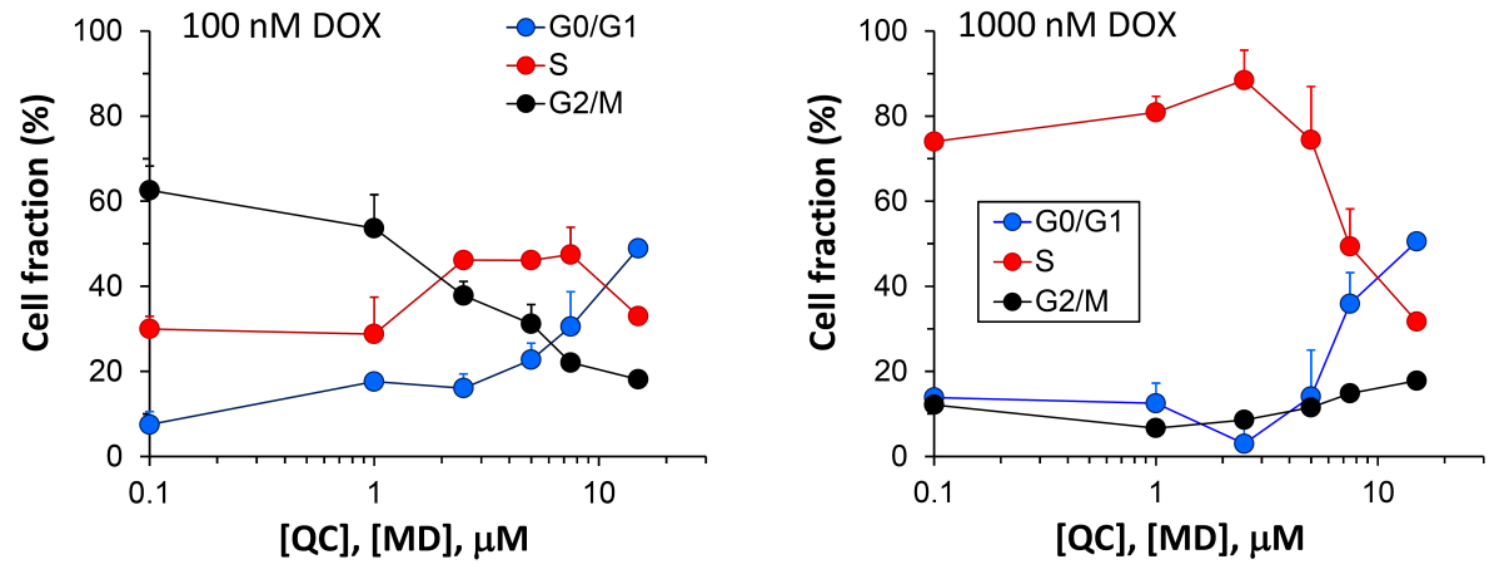

Fig. 2. Cell cycle distribution of Jurkat cells exposed for $18 \mathrm{~h}$ to $100 \mathrm{nM}$ (left) or $1000 \mathrm{nM}$ (right) DOX, in the presence of equimolar QC/MD combinations at indicated doses.

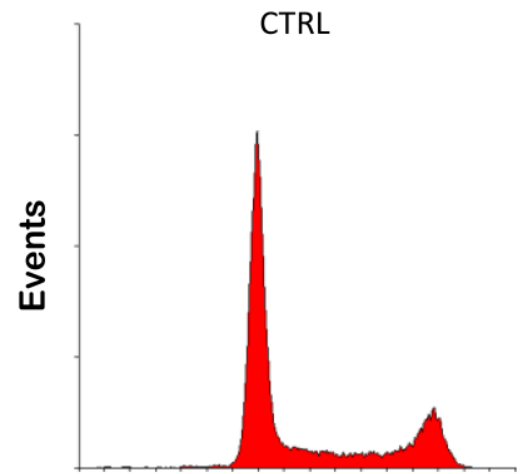

DNA content

$100 \mathrm{nM} \mathrm{DOX}+$ $7.5 \mu \mathrm{M} \mathrm{QC}+7.5 \mu \mathrm{M} \mathrm{MD}$

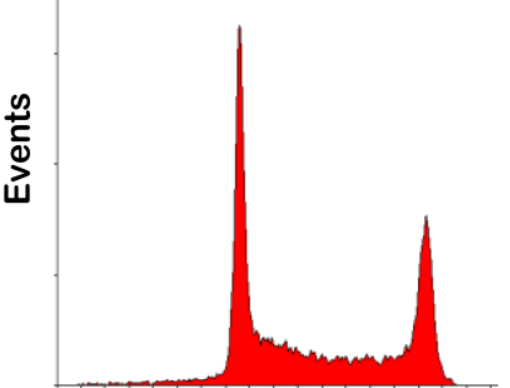

DNA content

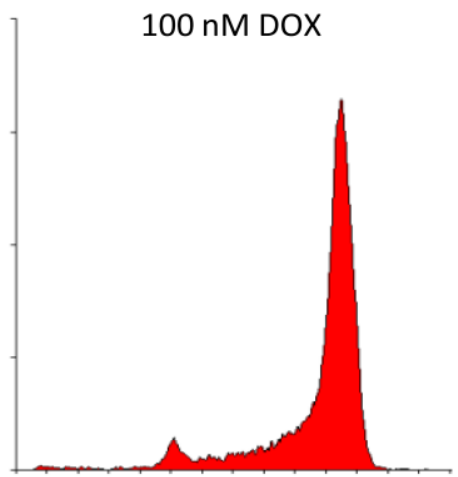

DNA content

$100 \mathrm{nM}$ DOX + $1 \mu \mathrm{M} Q \mathrm{C}+1 \mu \mathrm{M} \mathrm{MD}$

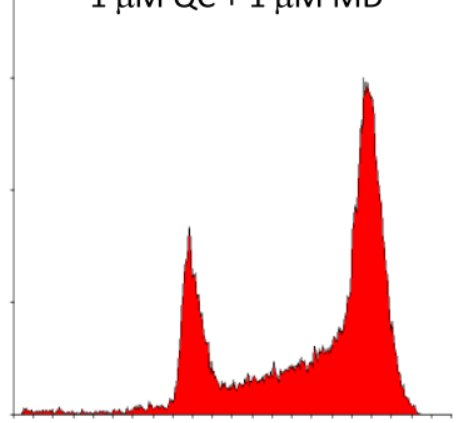

DNA content

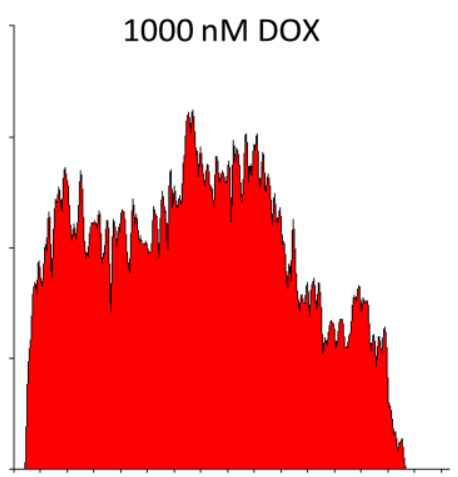

DNA content

$1000 \mathrm{nM}$ DOX + $5 \mu \mathrm{M} Q \mathrm{Q}+5 \mu \mathrm{M} \mathrm{MD}$

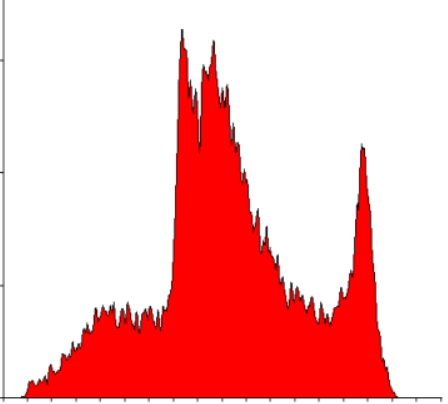

DNA content

Fig. 3. Histograms of cellular DNA content after $18 \mathrm{~h}$ treatments with indicated drugs. 
The cytotoxic effect of DOX was associated with apoptosis detected as phosphatidylserine externalization. High levels of DOX $(1000 \mathrm{nM})$ triggered a significant $44.9 \%$ apoptosis within $18 \mathrm{~h}$, but not within $6 \mathrm{~h}$ from exposure with only $3.7 \%$ (Fig. 4).

The $15 \mu \mathrm{M}$ equimolar QC/MD combination produced high apoptotic rates both at $6 \mathrm{~h}$ and $18 \mathrm{~h}$ after exposure with $37.5 \%$ respectively $69.6 \%$ apoptotic cells. Association with DOX enhanced apoptosis, with a 50.2\% dead cells after $6 \mathrm{~h}$ treatment and $79.6 \%$ after 18 $\mathrm{h}$ treatment.
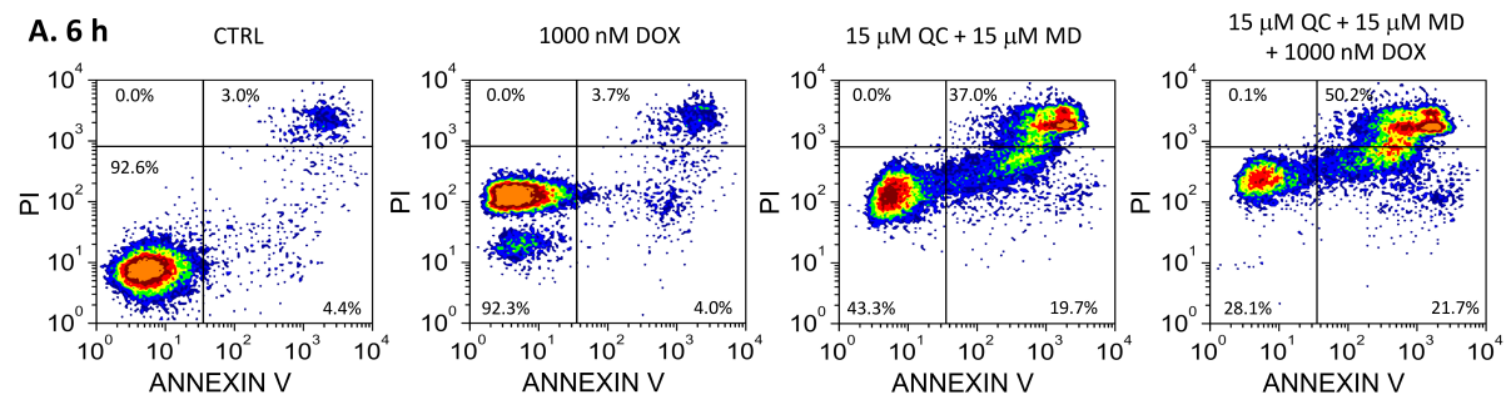

B. $18 \mathrm{~h}$
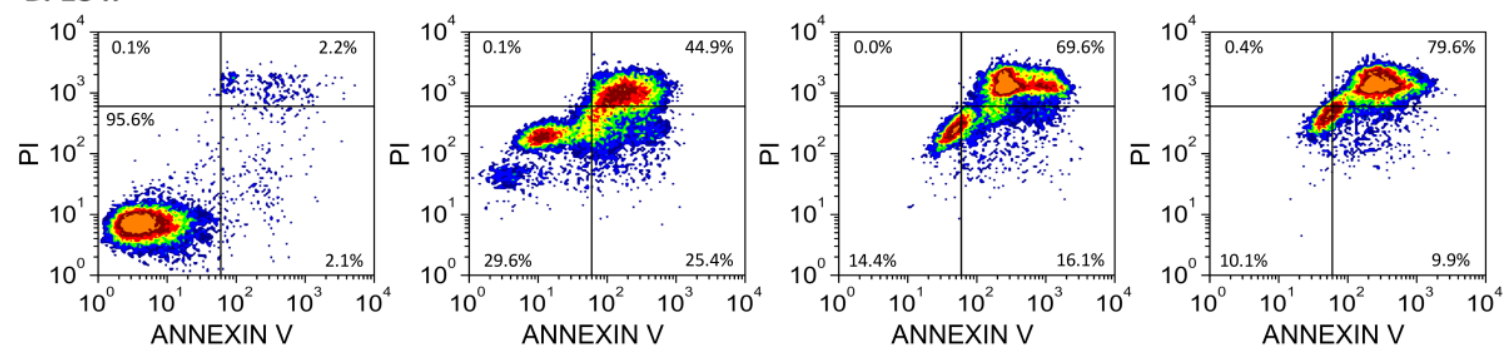

Fig. 4. Apoptosis induction determined by Annexin V/PI assay after exposure for $6 \mathrm{~h}(\mathrm{~A})$ or $18 \mathrm{~h}(\mathrm{~B})$ to indicated drugs.

\section{Conclusions}

Current data indicate that the inclusion of the QC:MD combination in doxorubicin-based treatment schemes for acute lymphoblastic leukemia could improve significantly the efficiency of the therapeutic drug.

The association promotes oxidative stress generation, in a dose dependent manner.

Quercetin exhibits both an oxidant and an antioxidant effect, with a primarily antioxidant effect on healthy cells and a greater susceptibility for it's pro-oxidant effect in neoplastic cells, and thus could prevent healthy cell injury during doxorubicin treatment as well as a protective agent against doxorubicin-induced cardiac toxicity.

The primary mechanisms responsible for the cytotoxic effect of doxorubicin appears to be oxidative stress generation, with release oxygen reactive species.

Cell cycle arrest due to doxorubicin exposure is determined by topoisomerase II inhibition and oxidative DNA injury, at G2/M phase.

$\mathrm{S}$ phase arrest seen at higher doxorubicin doses is probably due to DNA breakage and ATM (ataxia telangiectasia mutated) activation.

The high doses of doxorubicin also induces necrosis with a pre G0 peak. 
Association of Qc/MD induces cell cycle arrest at a $\mathrm{G} 0 / \mathrm{G} 1$ phase.This effect is probably determined by cell injury due to a higher oxidative-stress levels, leading to cellular death. Doxorubicin induces apoptosis and oxidative stress in a dose-dependent manner.

The cytotoxic effects of the association between DOX and QC/MD at clinically relevant levels appear to be additive, which could allow a reduction of the therapeutic dose of doxorubicin.

\section{Acknowledgments.}

This work was supported by a grant of the Romanian National Authority for Scientific Research, CNCS - UEFISCDI, project number PN-II-ID-PCE-2011-3-0800. Work for R.G.S., O.E.B., V.C. and M.T.I. was supported by four Young Researchers Fellowships of the Romanian Ministry of Education, UEFISCDI, project numbers 6/2014, 8/2015, $6 / 2015$ and $7 / 2015$, respectively.

\section{References:}

[1] Baran I, Cell proliferation versus apoptosis. Mechanisms and particularities under genotoxic or oxidative stress conditions. "Carol Davila" University Publishing House, Bucharest, 2014

[2] Murakami A,Ashida H, Terao J. Multitargeted cancer prevention by quercetin. Cancer Lett 2008;269:315-25

[2] Baran I, Ganea C, Privitera S, Scordino A, Barresi V, Musumeci F, et. al. Detailed analysis of apoptosis and delayed luminescence of human leukemia Jurkat $\mathrm{T}$ cells after proton-irradiation and treatments with oxidant agents and flavonoids. Oxid Med Cell Longev. 2012;2012:498914. doi:10.1155/2012/498914

[3] Baran I, Ionescu D, Filippi A, Mocanu M.M., Iftime A., Babes R., Tofolean I.T., Irimia R., Goicea A., Popescu V., Dimancea A., Neagu A., Ganea C., 2014. Novel insights into the antiproliferative effects and synergism of quercetin and menadione in human leukemia Jurkat T cells. Leuk Res. 2014;38(7):836-49.

[4] De Marchi U, Biasutto L, Garbisa S, Toninello A, Zoratti M. Quercetin can act either as an inhibitor or an inducer of the mitochondrial permeability transition pore: a demonstration of the ambivalent redox character of polyphenols. Biochim Biophys Acta. 2009;1787(12):1425-32.

[5] Baran I, Ionescu D, Privitera S, Scordino A, Mocanu MM, Musumeci F, Grasso R, Gulino M, Iftime A, Tofolean IT, Garaiman A, Goicea A, Irimia R, Dimancea A, Ganea C. Mitochondrial respiratory Complex I probed by delayed luminescence spectroscopy. JBO numele complet 2013;18(12):127006.

[6] http://chemoth.com/types/antracyclines

[7]Jeong JH, An Jy, Kwon YT, Rhee JG, Lee YJ.Effects of low dose quercetin:cancer specific inhibition of cell cycle progression.J Cell Biochem 2009;106:73-82

[8] Yen GC, Duh PD, Tsai HL, Huang SL. Pro-oxidative properties of flavonoids in human lymphocytes.Biosci Biotechnol Biochem 2003;67:1215-22

[9] Du G, Lin H, Wang M et al.Quercetin greatly improved therapeutic index of doxorubicin against $4 \mathrm{~T} 1$ brest cancer by its apposite effect on HIF-1 $\alpha$ in tumor and normal cells.Cancer Chemother Pharmacol 2010;65:277-87 
[10]Wang G,Zhang J, Liu L,Sharma S,Dong Q, Quercetin potentiates doxorubicin mediated antitumor effects against liver cancer through p53/Bcl-xl.PLos One 2012;7:e51764

[11]Thorn C,Oshiro C,Altman R.B.Doxorubicin pathways:pharmacodynamics and adverse effects,Pharmacogenics and genomics [12]http://elifesciences.org/content/elife/1/e00387.full.pdf

[13]Denard B,Lee C,Ye J 2012.Doxorubicin blocks proliferation of cancer cells through proteolytic activation of CREB3L1.eLife 1 e00090

[14] Baran I. et al., Cell Biochem. Biophys. 2010; 58:169-179

[15] Baran I. et al., Leukemia Res. 2014; 38:836-849

[16] Belizario JE, Alves J, Occhiucci JM, Garay-Malpartida M, Sesso A. A mechanistic view of mitochondrial death decision pores. Brazilian Journal of Medical and Biological Research. 2007;40:1011-24.

[17] Gibellini L, Pinti M, Nasi M, Montagna JP, De Biasi S, Roat E, Bertoncelli L, Cooper EL, Cossarizza A. Quercetin and cancer chemoprevention. Evid Based Complement Alternat Med. 2011;2011:591356.

[18] Ferry DR,Smith A,Malkhandi J, at al. Phase 1 clinical trial of the flavonoid quercetin: pharmacokinetics and evidence for in vitro tyrosine kinase inhibition.Clin Cancer Res 1996;2:659-68

[19] Am J Clin Nutr Oct 1, 2001, vol 74, no 4 418-425

[20] Rice s.a., 2003; Loke s.a., 2008; Kanadaswami s.a., 2005 